\title{
Breastfeeding in China: A Review of Changes in the Past Decade
}

\author{
Qing Li ${ }^{1}\left(\mathbb{D}\right.$, Jianli Tian ${ }^{1, *(\mathbb{D})}$, Fenglian $\mathrm{Xu}^{2}$ and Colin Binns ${ }^{3, * \mathbb{C}}$ \\ 1 Department of Nursing, Chengde Medical University, Chengde 067000 China; liqing8168@cdmc.edu.cn \\ 2 Data Analysis \& Surgical Outcomes Unit, Royal North Shore Hospital, Sydney, NSW 2065, Australia; \\ Fenglian.Xu@health.nsw.gov.au \\ 3 School of Public Health, Curtin University, Perth, WA 6845, Australia \\ * Correspondence: tjl@cdmc.edu.cn (J.T.); c.binns@curtin.edu.au (C.B.); Tel.: +61-892-662-950
}

Received: 25 September 2020; Accepted: 4 November 2020; Published: 7 November 2020

check for updates

\begin{abstract}
This review summarizes breastfeeding rates in China reported during the decade 2007-2018, a decade on from our previous review published in 2007. Compared with the studies undertaken before 2007 in China, recent studies are more likely to report breastfeeding rates using longer periods of observation, enabling rates to be summarized to six and 12 months postpartum in this review. There appears to have been a modest increase in breastfeeding in China. The mean duration of "any breastfeeding" was 10 months (9 to 11 months in the majority of cities), an increase compared with the previous review in which the mean of "any breastfeeding" duration was 8 months $(7$ to 9 months in the majority of cities). Using data from cohort studies, the proportion of infants being breastfed at 4 months increased from $78 \%$ in the earlier decade to $83 \%$ more recently. A second baby is usually breastfed for longer than the first, considering both "any" and "exclusive breastfeeding". China is a huge country and there is considerable diversity in culture, level of economic development, education and breastfeeding rates in different areas of China, but our review suggests that there has been some improvement in the "any breastfeeding" rate in the most recent decade.
\end{abstract}

Keywords: breastfeeding; prevalence; duration; review; China

\section{Introduction}

Breastfeeding $(\mathrm{BF})$ provides for optimal nutrition and development parameters, including improved functioning of the immune system, for infants until about six months of age and then into adult life [1,2]. The WHO (World Health Organization) and UNICEF (The United Nations Children's Fund) recommend that babies are breastfed exclusively to around six months and continue to be breastfed after the introduction of complementary food [3]. Increasing "exclusive breastfeeding" in the first six months of life to at least $50 \%$ across the globe is included in the WHO Nutrition goals for 2025 [4]. A new target set in the National Program of Action for Child Development in China from 2011 to 2020 is an "exclusive breastfeeding" rate of $50 \%$ and over at the sixth month of life. This is more specific than the previous target from 2001 to 2010, which simply stated a breastfeeding rate of $85 \%$, without specifying exclusivity [5].

We published a literature review in 2009 that summarized breastfeeding rates, duration and reasons of discontinuation of breastfeeding in China, including information on an increase of breastfeeding rates as a result of initiatives introduced to promote breastfeeding beginning in the 1990s [6]. Few cities and provinces reached the national target of an "exclusive breastfeeding" rate of $80 \%$ at four months after birth by 2000 [6]. Since 2007, more breastfeeding initiatives and interventions have been taken to promote breastfeeding in China. Maternity leave has been increased from 90 days to 120-180 days in 
recent years. The "universal two-child policy" has also been implemented throughout the country since 2016. It is time to review the changes of breastfeeding in China for the last decade.

The aim of this review is to document breastfeeding rates in China in the decade since 2007, including the changes of breastfeeding rates, duration and reasons for discontinuing breastfeeding. This review will update our previous review.

\section{Materials and Methods}

A literature search was undertaken using the Chinese databases: China National Knowledge Infrastructure (CNKI) and Wan Fang Medical Data and the English databases: Medline, Science Direct and ProQuest from 2007 to 2017. The databases were searched using the following key words: China, breastfeeding, breast feeding, breast-feeding and infant feeding. As shown in Figure 1, a total of 2894 studies were obtained, and ultimately 91 studies (including 49 cohort studies and 42 other studies) were analysed for this review. We assess the methodological quality of the selected studies based on the criteria proposed in Strengthening the Reporting of Observational Studies in Epidemiology (STROBE) [7] and the requirements of this study. All studies included met the following criteria: (1) sample size greater than 200; (2) loss to follow-up or incomplete records less than $20 \%$; (3) for prospective studies, more than three follow-up interviews with 1-2 month intervals; (4) breastfeeding rates calculated using correct definitions and statistical methods; and (5) published in peer-reviewed journals.

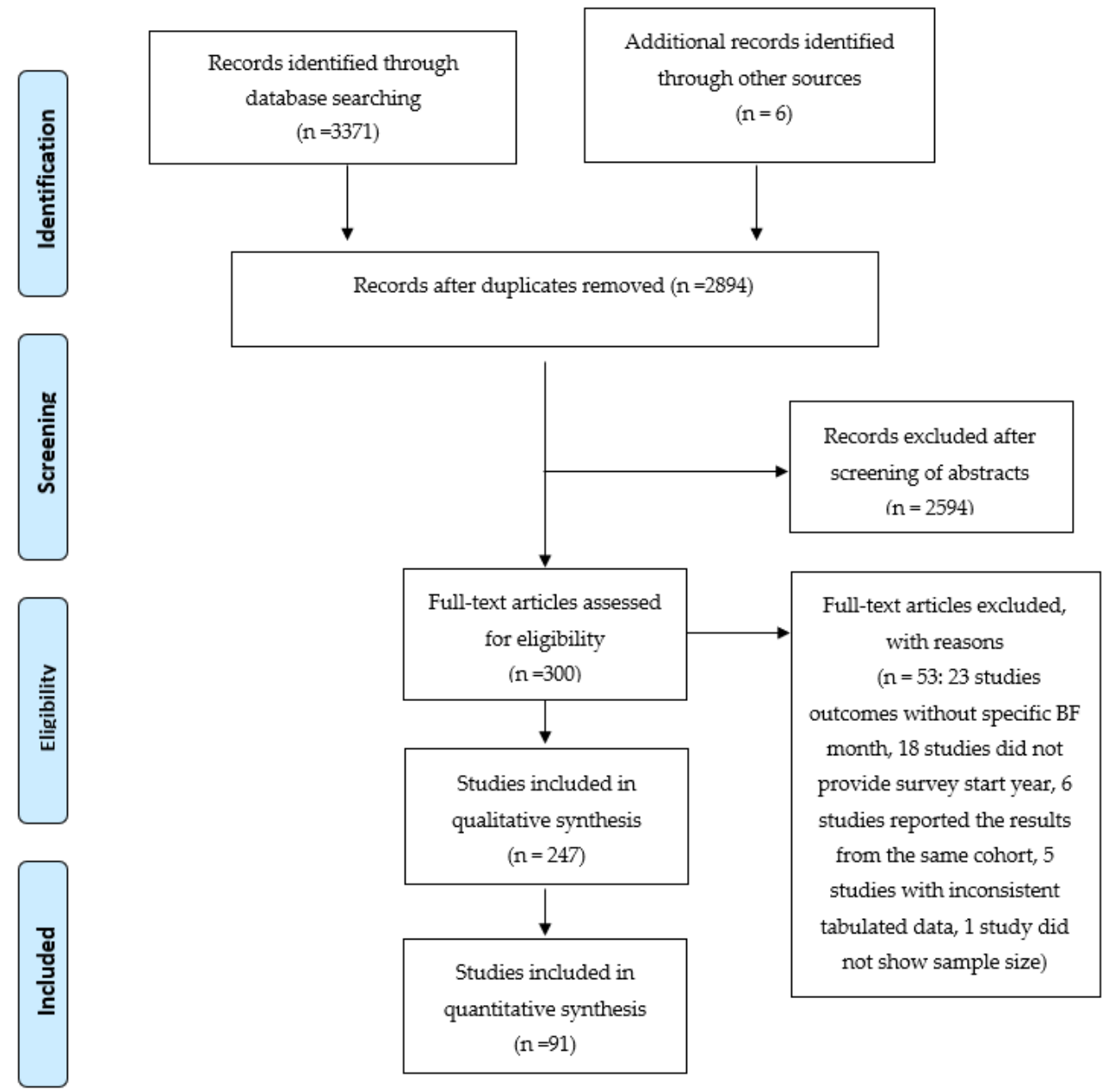

Figure 1. Flow diagram of systematic review following PRISMA protocol. 
All of the studies were assessed for quality using a method based on the STROBE criteria and used by us in a previous meta-analysis in China [8]. This method allocates a maximum score of 18, and papers that met a minimum score of 11 were included.

\section{Definitions of Breastfeeding}

WHO breastfeeding definitions are used in this paper [9].

"Exclusive breastfeeding": Breastfeeding while giving no other food or liquid, not even water, with the exception of drops or syrups consisting of vitamins, mineral supplements or medicines.

"Full breastfeeding": Exclusive breastfeeding or predominant breastfeeding or almost "exclusive breastfeeding". Breastmilk is the only source of milk given to the infant regardless of supplementation with other fluids such as water and orange juice.

"Any breastfeeding": The child has received breastmilk (direct from the breast or expressed) with or without other drink, formula or other infant food. "Any breastfeeding" included "exclusive breastfeeding" and "full breastfeeding" and "partial breastfeeding".

In some papers, only the term "breastfeeding" was used. The "breastfeeding" is categorized as "any breastfeeding" in this review.

\section{Results}

\subsection{Breastfeeding Rates in China from Cohort Studies}

Table 1 summarizes "exclusive breastfeeding" and "any breastfeeding" at six months in seven large, nine medium-sized and four small cities in China. The cities included in Table 1 are Beijing [10], Shanghai [11], Chengdu [12,13], Yinchuan [14], Changsha [15], Xi'an [16], Guangzhou [17], Ka'shen [18], Jinzhou [19], Shenzhen [20], Ake'su [21], Luzhou [22], Jiangyou [23], Ma'anshan [24], Mianyang [25], Ningbo [26], Wenling [27], Lishui [28], Yongkang [29] and Longnan [30]. The results are from 21 papers in Chinese and English published from 2007 to 2017 (Table 1).

The cohort studies in Table 1 were followed-up to six months and longer in 17 cities and to four months and over in three cities. Breastfeeding rates at six months from cohort studies are shown in Figure 2.

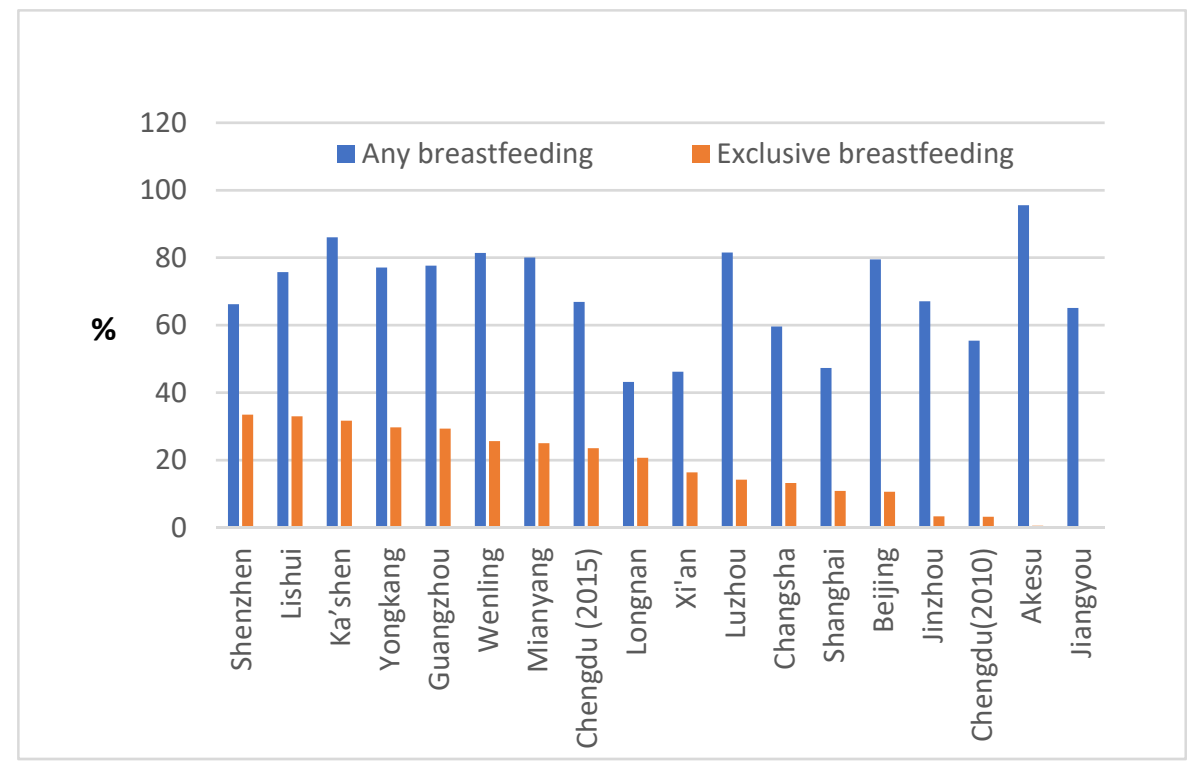

Figure 2. Breastfeeding rates (\%) at six months from cohort studies (2005-2016), China. 
Table 1. Breastfeeding rates (\%) in the first six months of life from cohort studies, China, 2007-2017.

\begin{tabular}{|c|c|c|c|c|c|c|c|}
\hline \multirow{3}{*}{$\begin{array}{c}\text { Study Details } \\
\text { Beijing [10] } \\
n=360\end{array}$} & \multirow{2}{*}{$\frac{\text { Baby's Age (month }{ }^{\text {a }} \text { ) }}{1}$} & \multirow{2}{*}{$\begin{array}{c}\text { Any Breastfeeding } \\
95.00\end{array}$} & \multicolumn{2}{|c|}{$95 \% \mathrm{CI}$} & \multirow{2}{*}{$\begin{array}{c}\text { Exclusive Breastfeeding } \\
64.17\end{array}$} & \multicolumn{2}{|c|}{$95 \% \mathrm{CI}$} \\
\hline & & & 92.75 & 97.25 & & 59.22 & 69.12 \\
\hline & 4 & 89.72 & 86.58 & 92.86 & 40.83 & 35.75 & 45.91 \\
\hline Survey year: 2007-2009 & 6 & 79.44 & 75.27 & 83.61 & 10.56 & 7.39 & 13.73 \\
\hline \multirow{4}{*}{$\begin{array}{c}\text { Shanghai [11] } \\
\quad n=296 \\
\text { Survey year: } 2014\end{array}$} & 0 & 82.09 & 77.72 & 86.46 & 28.72 & 23.57 & 33.87 \\
\hline & 1 & 77.03 & 72.24 & 81.82 & 27.70 & 22.60 & 32.80 \\
\hline & 3 & 66.22 & 60.83 & 71.61 & 24.32 & 19.43 & 29.21 \\
\hline & 6 & 47.30 & 41.61 & 52.99 & 10.81 & 7.27 & 14.35 \\
\hline \multirow{4}{*}{$\begin{array}{c}\text { Chengdu [12] } \\
n=438 \\
\text { Survey year: 2015-2016 }\end{array}$} & 0 & 87.90 & 84.85 & 90.95 & 60.96 & 56.39 & 65.53 \\
\hline & 1 & 86.07 & 82.83 & 89.31 & 47.95 & 43.27 & 52.63 \\
\hline & 4 & 79.45 & 75.67 & 83.23 & 37.90 & 33.36 & 42.44 \\
\hline & 6 & 66.89 & 62.48 & 71.30 & 23.52 & 19.55 & 27.49 \\
\hline \multirow{3}{*}{$\begin{array}{c}\text { Chengdu [13] } \\
n=760 \\
\text { Survey year: } 2010-2012\end{array}$} & 1 & 88.00 & 85.69 & 90.31 & $60.50^{b}$ & 57.02 & 63.98 \\
\hline & 3 & 73.40 & 70.26 & 76.54 & $52.90^{\mathrm{b}}$ & 49.35 & 56.45 \\
\hline & 6 & 55.40 & 51.87 & 58.93 & $3.20^{\mathrm{b}}$ & 1.95 & 4.45 \\
\hline \multirow{4}{*}{$\begin{array}{c}\text { Yinchuan [14] } \\
n=747 \\
\text { Survey year: 2014-2015 }\end{array}$} & 1 & 94.78 & 93.18 & 96.38 & 54.89 & 51.32 & 58.46 \\
\hline & 2 & 92.90 & 91.06 & 94.74 & 51.14 & 47.56 & 54.72 \\
\hline & 3 & 83.27 & 80.59 & 85.95 & 35.48 & 32.05 & 38.91 \\
\hline & 4 & 80.59 & 77.75 & 83.43 & 27.31 & 24.11 & 30.51 \\
\hline \multirow{4}{*}{$\begin{array}{c}\text { Changsha [15] } \\
\quad n=228 \\
\text { Survey year: } 2012\end{array}$} & 0 & 92.11 & 88.61 & 95.61 & 71.05 & 65.16 & 76.94 \\
\hline & 2 & 88.60 & 84.47 & 92.73 & 63.16 & 56.90 & 69.42 \\
\hline & 4 & 77.60 & 72.22 & 83.04 & 46.05 & 39.58 & 52.52 \\
\hline & 6 & 59.60 & 53.28 & 66.02 & 13.16 & 8.77 & 17.55 \\
\hline Xi'an [16] & 1 & 94.80 & 94.07 & 95.53 & 76.51 & 75.12 & 77.90 \\
\hline$n=3580$ & 3 & 75.20 & 73.79 & 76.61 & 47.09 & 45.45 & 48.73 \\
\hline Survey year: 2013 & 6 & 46.20 & 44.57 & 47.83 & 16.31 & 15.10 & 17.52 \\
\hline Guangzhou [17] & 1 & 97.60 & 96.13 & 99.07 & 72.12 & 67.81 & 76.43 \\
\hline$n=416$ & 3 & 97.12 & 95.51 & 98.73 & 71.39 & 67.05 & 75.73 \\
\hline Survey year: 2005-2006 & 6 & 77.64 & 73.64 & 81.64 & 29.33 & 24.95 & 33.71 \\
\hline
\end{tabular}


Table 1. Cont.

\begin{tabular}{|c|c|c|c|c|c|c|c|}
\hline \multirow{3}{*}{$\begin{array}{c}\text { Study Details } \\
\text { Ka'shen [18] } \\
n=300\end{array}$} & \multirow{2}{*}{$\frac{\text { Baby's Age (month }{ }^{\text {a) }}}{1}$} & \multirow{2}{*}{$\begin{array}{c}\text { Any Breastfeeding } \\
88.67\end{array}$} & \multicolumn{2}{|c|}{$95 \% \mathrm{CI}$} & \multirow{2}{*}{$\begin{array}{c}\text { Exclusive Breastfeeding } \\
51.33\end{array}$} & \multicolumn{2}{|c|}{$95 \% \mathrm{CI}$} \\
\hline & & & 85.08 & 92.26 & & 45.67 & 56.99 \\
\hline & 3 & 86.67 & 82.82 & 90.52 & 38.00 & 32.51 & 43.49 \\
\hline Survey year: 2015 & 6 & 86.00 & 82.07 & 89.93 & 31.67 & 26.41 & 36.93 \\
\hline \multirow{4}{*}{$\begin{array}{c}\text { Jinzhou [19] } \\
\quad n=972 \\
\text { Survey year: } 2014\end{array}$} & 2 & 83.13 & 80.78 & 85.48 & 49.07 & 45.93 & 52.21 \\
\hline & 3 & 80.86 & 78.39 & 83.33 & 49.07 & 45.93 & 52.21 \\
\hline & 4 & 77.06 & 74.42 & 79.70 & 30.56 & 27.66 & 33.46 \\
\hline & 6 & 67.08 & 64.13 & 70.03 & 3.29 & 2.17 & 4.41 \\
\hline \multirow{3}{*}{$\begin{array}{c}\text { Shenzhen [20] } \\
\quad n=325 \\
\text { Survey year: } 2013\end{array}$} & 1 & 90.15 & 86.91 & 93.39 & 87.38 & 83.77 & 90.99 \\
\hline & 4 & 82.71 & 78.39 & 87.03 & 59.66 & 54.06 & 65.26 \\
\hline & 6 & 66.20 & 60.70 & 71.70 & 33.45 & 27.96 & 38.94 \\
\hline \multirow{4}{*}{$\begin{array}{c}\text { Akesu [21] } \\
n=400 \\
\text { Survey year: } 2011-2012\end{array}$} & 0 & 99.50 & 98.81 & 100.19 & 96.00 & 94.08 & 97.92 \\
\hline & 1 & 99.00 & 98.02 & 99.98 & 79.00 & 75.01 & 82.99 \\
\hline & 3 & 98.25 & 96.96 & 99.54 & 62.50 & 57.76 & 67.24 \\
\hline & 6 & 95.50 & 93.47 & 97.53 & 0.50 & 0.14 & 1.80 \\
\hline \multirow{3}{*}{$\begin{array}{c}\text { Luzhou [22] } \\
n=486 \\
\text { Survey year: } 2012\end{array}$} & 1 & 92.59 & 90.26 & 94.92 & 45.06 & 40.64 & 49.48 \\
\hline & 3 & 89.71 & 87.01 & 92.41 & 42.80 & 38.40 & 47.20 \\
\hline & 6 & 81.48 & 78.03 & 84.93 & 14.2 & 11.10 & 17.30 \\
\hline \multirow{4}{*}{$\begin{array}{c}\text { Jiangyou [23] } \\
n=695 \\
\text { Survey year: 2010-2011 }\end{array}$} & 0 & 95.10 & 93.40 & 96.70 & - & - & - \\
\hline & 1 & 92.70 & 90.70 & 94.70 & - & - & - \\
\hline & 3 & 85.10 & 82.30 & 87.80 & - & - & - \\
\hline & 6 & 65.10 & 61.20 & 69.00 & - & - & - \\
\hline \multirow{3}{*}{$\begin{array}{c}\text { Ma'anshan [24] } \\
\quad n=343 \\
\text { Survey year: } 2009\end{array}$} & 2 & 88.92 & 85.60 & 92.24 & 57.43 & 52.20 & 62.66 \\
\hline & 3 & 84.84 & 81.04 & 88.64 & 55.98 & 50.73 & 61.23 \\
\hline & 4 & 81.92 & 77.85 & 85.99 & 51.60 & 46.31 & 56.89 \\
\hline \multirow{3}{*}{$\begin{array}{c}\text { Mianyang [25] } \\
n=1532 \\
\text { Survey year: } 2008\end{array}$} & 1 & 95.04 & 93.95 & 96.13 & 91.06 & 89.63 & 92.49 \\
\hline & 4 & 87.79 & 86.15 & 89.43 & 45.82 & 43.32 & 48.32 \\
\hline & 6 & 80.03 & 78.03 & 82.03 & 25.00 & 22.83 & 27.17 \\
\hline
\end{tabular}


Table 1. Cont.

\begin{tabular}{|c|c|c|c|c|c|c|c|}
\hline \multirow{4}{*}{$\begin{array}{c}\text { Study Details } \\
\text { Ningbo [26] } \\
n=318\end{array}$} & \multirow{2}{*}{$\frac{\text { Baby's Age (month }{ }^{\mathrm{a}} \text { ) }}{2}$} & \multirow{2}{*}{$\begin{array}{c}\text { Any Breastfeeding } \\
82.39\end{array}$} & \multicolumn{2}{|c|}{$95 \% \mathrm{CI}$} & \multirow{2}{*}{$\begin{array}{c}\text { Exclusive Breastfeeding } \\
36.00\end{array}$} & \multicolumn{2}{|c|}{$95 \% \mathrm{CI}$} \\
\hline & & & 78.20 & 86.58 & & 30.58 & 41.12 \\
\hline & 3 & 66.67 & 61.49 & 71.85 & 36.00 & 30.58 & 41.12 \\
\hline & 4 & 50.63 & 45.13 & 56.13 & 23.60 & 18.91 & 28.25 \\
\hline Survey year: 2008 & 5 & 47.80 & 42.31 & 53.29 & 10.00 & 13.8 & 13.30 \\
\hline \multirow{4}{*}{$\begin{array}{c}\text { Wenling [27] } \\
\quad n=500 \\
\text { Survey year: } 2014\end{array}$} & 0 & 91.80 & 89.40 & 94.20 & 80.20 & 76.71 & 83.69 \\
\hline & 1 & 91.00 & 88.49 & 93.51 & 70.20 & 66.19 & 74.21 \\
\hline & 4 & 86.00 & 82.96 & 89.04 & 52.60 & 48.22 & 56.98 \\
\hline & 6 & 81.40 & 77.99 & 84.81 & 25.60 & 21.77 & 29.43 \\
\hline Lishui [28] & 1 & 89.90 & 85.80 & 94.00 & 34.62 & 28.15 & 41.09 \\
\hline$n=208$ & 3 & 84.82 & 79.73 & 89.91 & 34.62 & 28.15 & 41.09 \\
\hline Survey year: 2014 & 6 & 75.68 & 69.50 & 81.86 & 32.97 & 26.20 & 39.74 \\
\hline Yongkang [29] & 1 & 92.50 & 90.50 & 94.50 & 78.11 & 74.97 & 81.25 \\
\hline$n=667$ & 3 & 89.06 & 86.69 & 91.43 & 58.92 & 55.19 & 62.65 \\
\hline Survey year: 2013 & 6 & 77.06 & 73.87 & 80.25 & 29.69 & 26.22 & 33.16 \\
\hline Longnan [30] & 1 & 93.13 & 90.87 & 95.39 & 78.75 & 75.09 & 82.41 \\
\hline$n=480$ & 3 & 68.13 & 63.96 & 72.30 & 42.29 & 37.87 & 46.71 \\
\hline Survey year: 2009 & 6 & 43.13 & 38.70 & 47.56 & 20.63 & 17.01 & 24.25 \\
\hline
\end{tabular}

a: 0 month refers to the time at discharge from hospital for birth, generally at one or two weeks after birth. ${ }^{\text {b}}$ : Full breastfeeding. Beijing: the capital city of China. Shanghai: large city, Eastern China. Chengdu: the capital city of Sichuan province, Southwest China. Yinchuan: the capital city of Ningxia Autonomous Region, Northwest China. Changsha: the capital city of Hunan province, South China. Xi' an: the capital city of Shaanxi province, Northwest China. Guangzhou: the capital city of Guangdong province, Southeast China. Ka'shen: a medium-sized city in Xinjiang Uygur Autonomous Region, West China. Jinzhou: a medium-sized city in Liaoning province, Northeast China. Shenzhen: a medium-sized city in Guangdong province, Southeast China. Akesu: a medium-sized city in Xinjiang Uygur Autonomous Region, West China. Luzhou: a medium-sized city in Sichuan province, Southwest China. Jiangyou: a medium-sized city in Sichuan province, Southwest China. Ma'anshan: a medium-sized city in Anhui province, Southeast China. Mianyang: a medium-sized city in Sichuan province, Southwest China. Ningbo: a medium-sized city in Zhejiang province, East China. Wenling: a small city in Zhejiang province, East China. Lishui: a small city in Zhejiang province, East China. Yongkang: a small city in Zhejiang province, East China. Longnan: a small city in Gansu province, Northwest China. 
Table 1 shows that the "any breastfeeding" rate at six months ranged from $43.13 \%$ to $95.50 \%$ in 17 cities (excluding Yingchuan, Ma'anshan and Ningbo where the follow-up time was less than six months). Of the 17 cities, the "any breastfeeding" rates at six months were above 85\% in AKesu and Ka'shen of Xinjiang Uygur Autonomous Region; between $80 \%$ and $81 \%$ in Luzhou and Mianyang of Sichuan province, and Wengling of Zhejiang province; and below $80 \%$ in other 12 cities. In majority cities $(15 / 17=88 \%)$, the "any breastfeeding" rates at sixth months were below $85 \%$.

Table 1 also shows that "exclusive breastfeeding" rates at six months were between $0.50 \%$ and $33.45 \%$ in 17 cities (excluding Yingchuan, Ma'anshan and Ningbo where the follow-up times were less than six months). The "exclusive breastfeeding" rates were below the target of $50 \%$ "exclusive breastfeeding" rate at six months [5]. In Akesu, a medium-sized city from Xinjiang, 96\% babies were exclusively breastfed before discharged from hospital for birth, but only $0.5 \%$ babies were still exclusively breastfed at six months after birth [21]. The low "exclusive breastfeeding" rate in Akesu was consistent with a report (based on a study in 2003-2004) from Shihezi, Xinjiang [31]. The average "exclusive breastfeeding" duration in Xinjiang was 1.8 months [32].

"Any breastfeeding" rates before discharge from hospitals were between $82.09 \%$ and $99.50 \%$ (reported from six studies in six cities in Table 1). "Exclusive breastfeeding" rates before discharge from hospitals showed a wide variation from $28.70 \%$ to $96.00 \%$ (reported from five studies in five cities in Table 1).

"Any breastfeeding" rates at the 12th month ranged from $10.97 \%$ to $73.26 \%$ [11,23,33-35] (from five studies in five cities in Table 2). Less than $1 \%(0.26-0.38 \%)$ of children were breastfed to two years [34,35].

Table 2. "Any breastfeeding" rates at 12 months after birth from cohort studies, China, 2010-2014.

\begin{tabular}{cccccc}
\hline Province or Big City & $\begin{array}{c}\text { Survey } \\
\text { Commencement }\end{array}$ & $\begin{array}{c}\text { Women } \\
(\boldsymbol{n})\end{array}$ & $\begin{array}{c}\text { Any BF Rate } \\
\mathbf{( \% )}\end{array}$ & 95\% CI \\
\hline Shaanxi [33] & 2014 & 1350 & 73.26 & 70.90 & 75.62 \\
Anhui [34] & 2012 & 1332 & 27.40 & 25.00 & 29.80 \\
Shanghai [11] & 2014 & 296 & 14.19 & 10.21 & 18.17 \\
Sichuan [23] & 2010 & 695 & 12.90 & 9.90 & 15.80 \\
Guangzhou [35] & 2013 & 383 & 10.97 & 7.84 & 14.10 \\
\hline \multicolumn{7}{c}{ BF, breastfeeding. }
\end{tabular}

In addition to the studies in Table 1 , a cohort study $(n=1350 ; 2013-2014)$ in rural areas from three cities in Shaanxi Province, showed that the "any breastfeeding" rates were $67.85 \%$ and $39.41 \%$ at six and 18 months, respectively, and the "exclusive breastfeeding" rate at six months was $35.04 \%$ [33]. Another cohort study from poor areas in Anhui province in 2012-2013 showed that the "any breastfeeding" rates were $75.45 \%, 71.02 \%, 27.4 \%$ and $0.38 \%$ at four, six, 12 and 24 months, respectively [34]. A study in Guangzhou (2013-2014) showed that "any breastfeeding" rates were $65.49 \%, 47.58 \%, 10.97 \%$ and $0.26 \%$ at four, six, 12 and 24 months, respectively [35]. A cohort study in Zhoushan, a medium-sized city in Zhejiang Province, showed that "full breastfeeding" rates were $87.3 \%$, $68.5 \%, 48.1 \%, 26.2 \%$ and $5.6 \%$ at one, three, six, nine and 12 months, respectively, in 2002-2015 [36]. A notable improvement in breastfeeding cohort studies in China was that follow-up time was extended to six months in more studies compared with those before 2007 [6].

The breastfeeding rates from three cities (Beijing, Guangzhou and Luzhou) were reported both in the previous literature review and the current review although the follow-up times were different. Compared with our previous literature review, "any breastfeeding" rates in Beijing increased significantly. For example, the "any breastfeeding" rate in Beijing was 95.00\% (95\% CI $=92.75-97.25)$ at one month and $89.72 \%$ (95\% CI = 86.58-92.86) at four months in 2007-2009 [10] compared to $84.00 \%$ (95\% CI $=76.8-91.2)$ and 76.00\% (95\% CI = 67.6-84.4) in 1997 [6]. "Exclusive breastfeeding" rates at one and four months in 2007-2009 in Beijing were not statistically different from those in 1997 [6,10]. "Any breastfeeding" rates at three months in Guangzhou also increased compared with our previous literature review from $93.2 \%(95 \% \mathrm{CI}=91.8-94.6)$ in $1998-1999$ [6] to $97.12 \%(95 \% \mathrm{CI}=95.51-98.73)$ 
in 2005-2006 [17]. The "exclusive breastfeeding" rate at one month in Guangzhou decreased to $72.12 \%(95 \% \mathrm{CI}=67.81-76.43)$ in $2005-2006$ [17] from $90.5 \%(95 \% \mathrm{CI}=88.9-92.1)$ in 1998-1999 [6]. "Any breastfeeding" rates at one month and "exclusive breastfeeding" rates at three months in Guangzhou in 2005-2006 were not statistically different compared with those in 1998-1999. On the other hand, in Luzhou, "any breastfeeding" rates at one month decreased [6,22]. The "any breastfeeding" rate at one month in Luzhou was 92.59\% (95\% CI $=90.26-94.92)$ in 2012 [22] and 97.5\% (95\% CI $=95.4-99.6)$ in 2002 [6]. The "exclusive breastfeeding" rates at one month and three months in Luzhou decreased significantly compared with previous literature review [6,22]. The "exclusive breastfeeding" rates in Luzhou were $45.06 \%(95 \% \mathrm{CI}=40.64-49.48)$ at one month and $42.8 \%(95 \% \mathrm{CI}=38.4-47.2)$ at three months in 2012 [22]; while the "exclusive breastfeeding" rates were $89.6 \%(95 \% \mathrm{CI}=85.4-93.8)$ at one month and $83.7 \%(95 \% \mathrm{CI}=78.6-88.8)$ at three months in 2002 [6].

Table 2 summarizes the "any breastfeeding" rate at 12 months from five cohort studies.

\subsection{Breastfeeding Rates in China from Other Types of Studies}

Table 3 summarizes "exclusive breastfeeding" and "any breastfeeding" rates at six months and breastfeeding initiation rates in ten large cities and four provinces in China. The cities and provinces included in Table 3 are Tianjin [37,38], Shanghai [39,40], Chongqing [41,42], Zhengzhou [43,44], Wuhan [45,46], Guangzhou [47,48], Changsha [49], Xining [50,51], Changchun [52,53], Nanchang [54], Zhejiang [55,56], Shanxi [57-59], Anhui [34,60,61] and Jiangsu [62,63]. For the provinces or cities where more than one study was carried out, the most recent results have been presented. The research methods included cross-sectional and retrospective studies and cohort studies that did not satisfy the criteria for Table 1.

Table 3 shows that breastfeeding initiation rates were between $77.02 \%$ and $98.00 \%$; "any breastfeeding" rates at six months were between $63.41 \%$ and $92.93 \%$; and "exclusive breastfeeding" rates at six months were between $17.87 \%$ and $58.50 \%$. Of the 10 cities and four provinces in Table 3, the exclusive breastfeeding rate at six months was above $50 \%$ (the national goal) in three cities (Wuhan, Shanghai and Guangzhou) $(3 / 14=21 \%)$ and the "any breastfeeding" rate at six months was above $85 \%$ (the previous national goal) in three cities and one province (Nanchang, Changchun, Chongqing and Shanxi) $(4 / 14=29 \%)$. The lower number reaching the "exclusive breastfeeding" target needs improving.

Furthermore, the studies in Table 3, in a cross-sectional study $(n=1288)$ from 32 maternity and child healthcare hospitals in China, the rate of "any breastfeeding" rate at discharge (in hospital) was $96.4 \%$ and the "exclusive breastfeeding" rate at discharge was $46.6 \%$ in 2010 [64]. A major survey $(n=14,262)$ of rural infants from 5 provinces in Western China in 2009 found that the "any breastfeeding" rate at discharge was $97.46 \%$ and the "exclusive breastfeeding" rate at four months was $52.02 \%$ in these rural areas [65]. A cross-sectional survey in 12 provinces in central and western China in 2010 showed that the overall "any breastfeeding" rate was $98.3 \%$, and the overall "exclusive breastfeeding" rate at six months was $28.7 \%$ [66]. A national representative survey $(n=14,458)$ from 55 counties in 30 provinces in China (2013) showed that the crude "exclusive breastfeeding" rate at six months of age was $20.73 \%$ (908/4381), and the weighted "exclusive breastfeeding" rate was $18.6 \%$ [67]. These studies showed that "exclusive breastfeeding" rates at six months were below 30\% (20.73-28.70\%).

A recent survey found that "exclusive breastfeeding" rates at six months in Beijing $(n=890)$ [68], Shanghai $(n=5672)$ [69] and Dalian $(n=32,466)$ [70] were $31.82 \%, 51.34 \%$ and $55.79 \%$, respectively. In Shenzhen, "exclusive" and "any breastfeeding" rates at six months were $61.2 \%$ and $91.3 \%$, respectively $(n=1000)$ in 2015 [71]. A survey $(n=1019)$ in nine community hospitals from three cities in Anhui province showed that the overall "exclusive breastfeeding" rate at six months were $61.9 \%$, including $64.5 \%$ in Hefei, the capital city of Anhui, $60.7 \%$ in Fuyang and 59.5\% in Wufu [72]. The "exclusive breastfeeding" rates reported in these recent studies (2016-2018) show improvement. This may be associated with the promotion of breastfeeding in recent years [68-72]. 
Table 3. Breastfeeding rates (\%) in China 2007-2018.

\begin{tabular}{|c|c|c|c|c|}
\hline Study Site & Initiation Rate & $\begin{array}{l}\text { Any Breastfeeding } \\
\text { Rate at Six Months }\end{array}$ & $\begin{array}{l}\text { Exclusive Breastfeeding } \\
\text { Rate at Six Months }\end{array}$ & Study Details \\
\hline Changchun * $[52,53]$ & 98.00 & 89.97 & 35.53 & $\begin{array}{l}\text { a. Cross-sectional study 2013, } n=349 ; \\
\text { b. Cohort study 2007-2010, } n=1600\end{array}$ \\
\hline Nanchang * [54] & $96.21^{\mathrm{a}}$ & 92.93 & 34.53 & Cohort study 2011-2013, $n=976$ \\
\hline Zhejiang ${ }^{\mathrm{P}}[55,56]$ & 95.80 & 80.00 & 40.89 & $\begin{array}{l}\text { a. Retrospective study 2015-2017, } \\
\qquad n=429 ; \\
\text { b. Cross-sectional study } 2013, n=675\end{array}$ \\
\hline Zhengzhou * $[43,44]$ & 95.26 & 82.50 & 26.25 & $\begin{array}{l}\text { a. Cross-sectional study 2011-2012, } \\
\qquad n=612 ; \\
\text { b. } 2013-2014, n=800\end{array}$ \\
\hline Shanxi P [57-59] & 93.75 & $86.45^{\mathrm{b}}$ & 29.78 & $\begin{array}{l}\text { a. Cross-sectional studies 2016, } \\
n=1193 \\
\text { b. } 2014, n=487 \\
\text { c. } 2009, n=240\end{array}$ \\
\hline Chongqing ** $[41,42]$ & 93.49 & 85.47 & 39.25 & $\begin{array}{l}\text { a. Cohort study } 2015-2016, n=215 \\
\text { b. } 2016-2018, n=57,382\end{array}$ \\
\hline Changsha * [49] & 92.20 & - & 40.00 & a. Cross-sectional study 2014, $n=1014$ \\
\hline Shanghai $* *[39,40]$ & 90.00 & - & 57.91 & $\begin{array}{l}\text { a. Cross-sectional study } 2016, n=200 ; \\
\text { b. Cohort study 2014, } n=815\end{array}$ \\
\hline Xining * $[50,51]$ & 89.80 & 63.41 & 19.51 & $\begin{array}{l}\text { a. Cross-sectional study 2012-2015, } \\
\qquad n=1148 ; \\
\text { b. Cohort study 2013, } n=287\end{array}$ \\
\hline Jiangsu P $[62,63]$ & 89.46 & 75.00 & 37.50 & $\begin{array}{l}\text { a. Cross-sectional study 2014-2015, } \\
\qquad n=320 \\
\text { b. Cohort study 2010-2013, } n=759\end{array}$ \\
\hline Tianjin ** $[37,38]$ & $84.60^{\mathrm{a}}$ & 82.00 & 48.00 & $\begin{array}{l}\text { a. Cross-sectional study } 2015, n=818 \\
\text { b. Cohort study 2011-2012, } n=200\end{array}$ \\
\hline Wuhan * $[45,46]$ & 83.75 & - & 58.50 & $\begin{array}{l}\text { a. Cross-sectional studies 2013-2015, } \\
\qquad \begin{array}{c}n=2000 \\
\text { b. } 2016-2017, n=494\end{array}\end{array}$ \\
\hline Guangzhou * $[47,48]$ & 77.90 & 79.24 & 57.12 & $\begin{array}{l}\text { a. Cross-sectional studies 2011-2014 } \\
\qquad n=1180 \\
\text { b. } 2013-2014, n=289\end{array}$ \\
\hline Anhui $\mathrm{P}[34,60,61]$ & 77.02 & 71.02 & $17.87^{\mathrm{c}}$ & $\begin{array}{l}\text { a. Cross-sectional studies 2008, } \\
\quad n=1736 \\
\text { b. Cohort study } 2012-2013, n=1332 ; \\
\text { c. } 2008-2010, n=2747\end{array}$ \\
\hline
\end{tabular}

a: At 1st month. ${ }^{\text {b: }}$ At 4-6 months. ${ }^{c}$ : Full breastfeeding; : Province; ${ }^{*}$ Capital city of province. ${ }^{* *}$ Large city (municipality directly under the Central Government). In the column "Study Details", where there is more than one study from the same city, they are labelled $a, b$ and $c$.

\subsection{Reasons for the Variation in Reported Breastfeeding Rates}

This review shows that breastfeeding rates from studies conducted in different cities and areas in China can show major differences. The large range in breastfeeding rates may be associated with differences in breastfeeding promotion strategies, study methods, study populations, culture and other factors including mother's age, educational background, household income, residential area, delivery mode and family support. For example, in cross-sectional or retrospective studies, the methods used to assess infant feeding might be in the "last $24 \mathrm{~h}$ prior to the survey" or "during the last month".

\subsection{Length of Breastfeeding and Proportion Breastfeeding in China from 2007 to 2017}

The mean duration of "any breastfeeding" between 2007 and 2017 was between eight and 12 months (9 to 11 months in the majority of cities) reported from cross sectional studies. [23,34,44,50,73-80]. The mean duration was 10.1 months (weighted by provincial population) from eight cross-sectional studies. In the previous study, the weighted mean duration of breastfeeding was 8.0 months from cross-sectional studies. The reported mean duration of breastfeeding shows an increase of almost two months compared with that in the previous review (7 to 9 months in majority cities) [6]. For example, "any breastfeeding" duration in Shanghai was 7.4 months in between 1999 and 2002 [6] and has 
increased to 8.45 and 9.98 months (Figures 3 and 4). The mean durations of "any breastfeeding" reported from studies before and after 2007 are shown in Figure 5. This shows that the majority of studies reporting longer durations of breastfeeding have been published since 2007.

The proportion of mothers breastfeeding at four months was reported in both decades in the cohort studies. The weighted (on provincial population) mean rate of four months "any breastfeeding" was $78.2 \%$ before 2007 and $83.0 \%$ more recently.

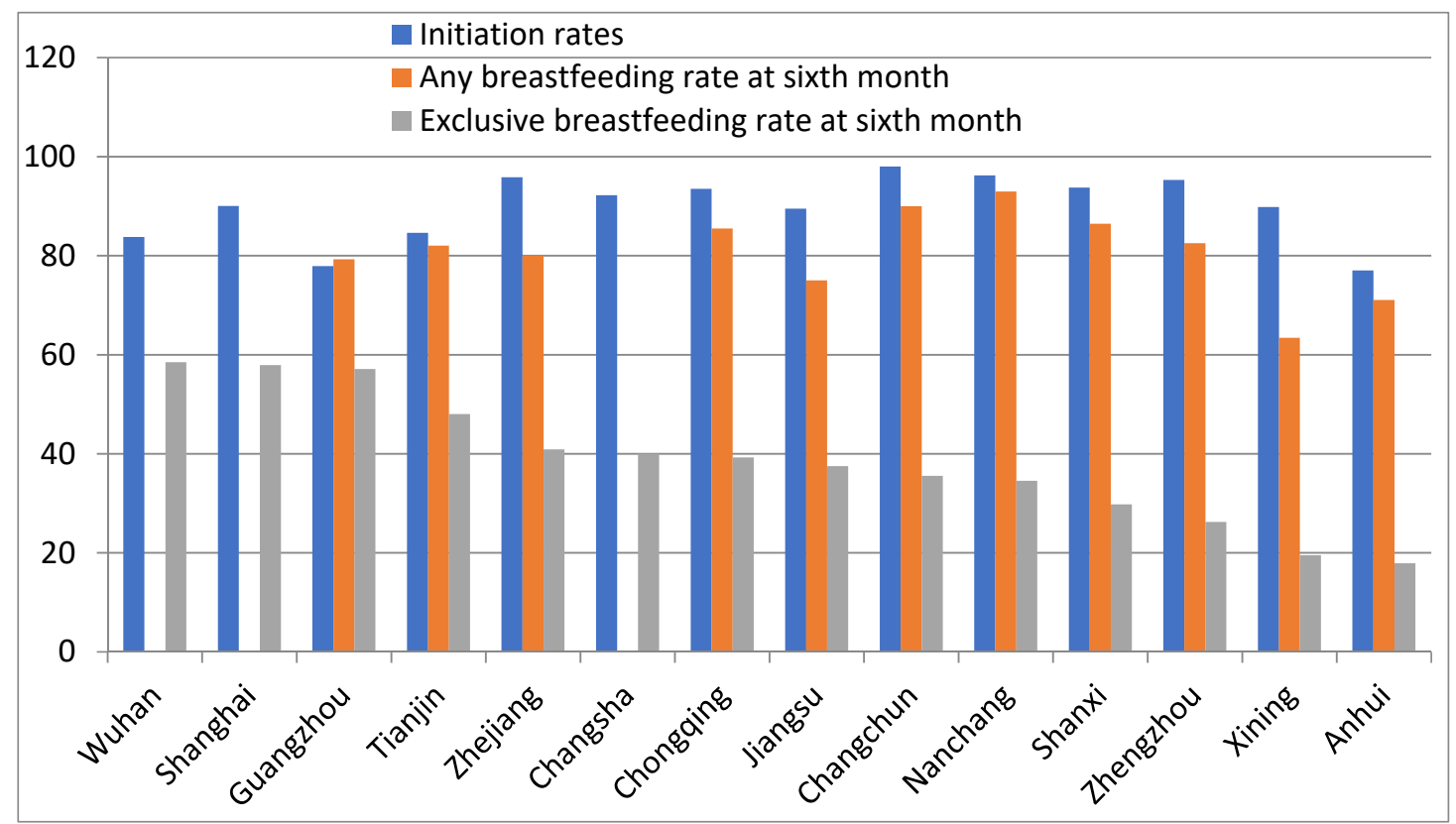

Figure 3. Breastfeeding in China from other studies, 2007-2018.

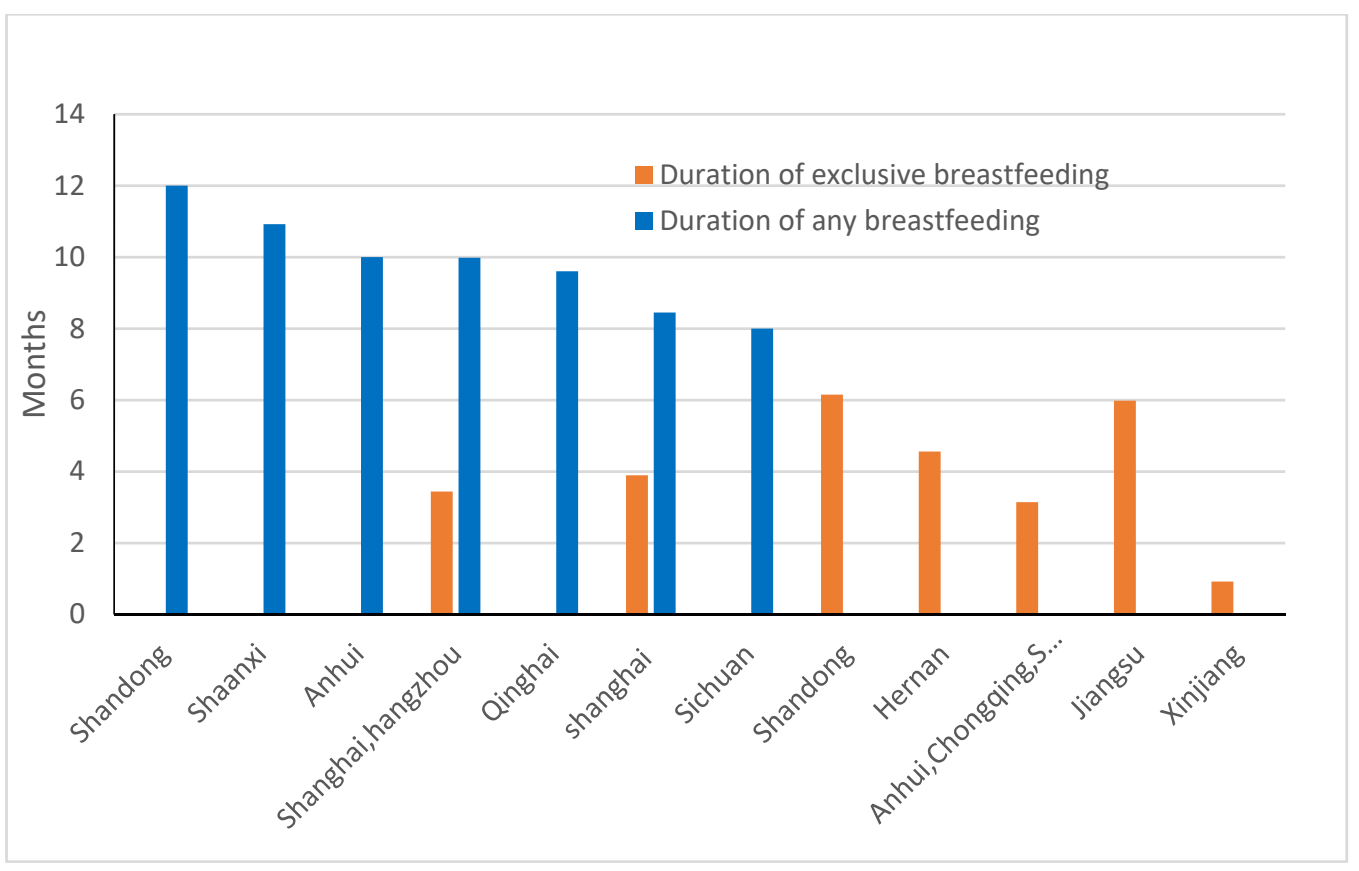

Figure 4. Reported length of breastfeeding in China, 2007-2017. 


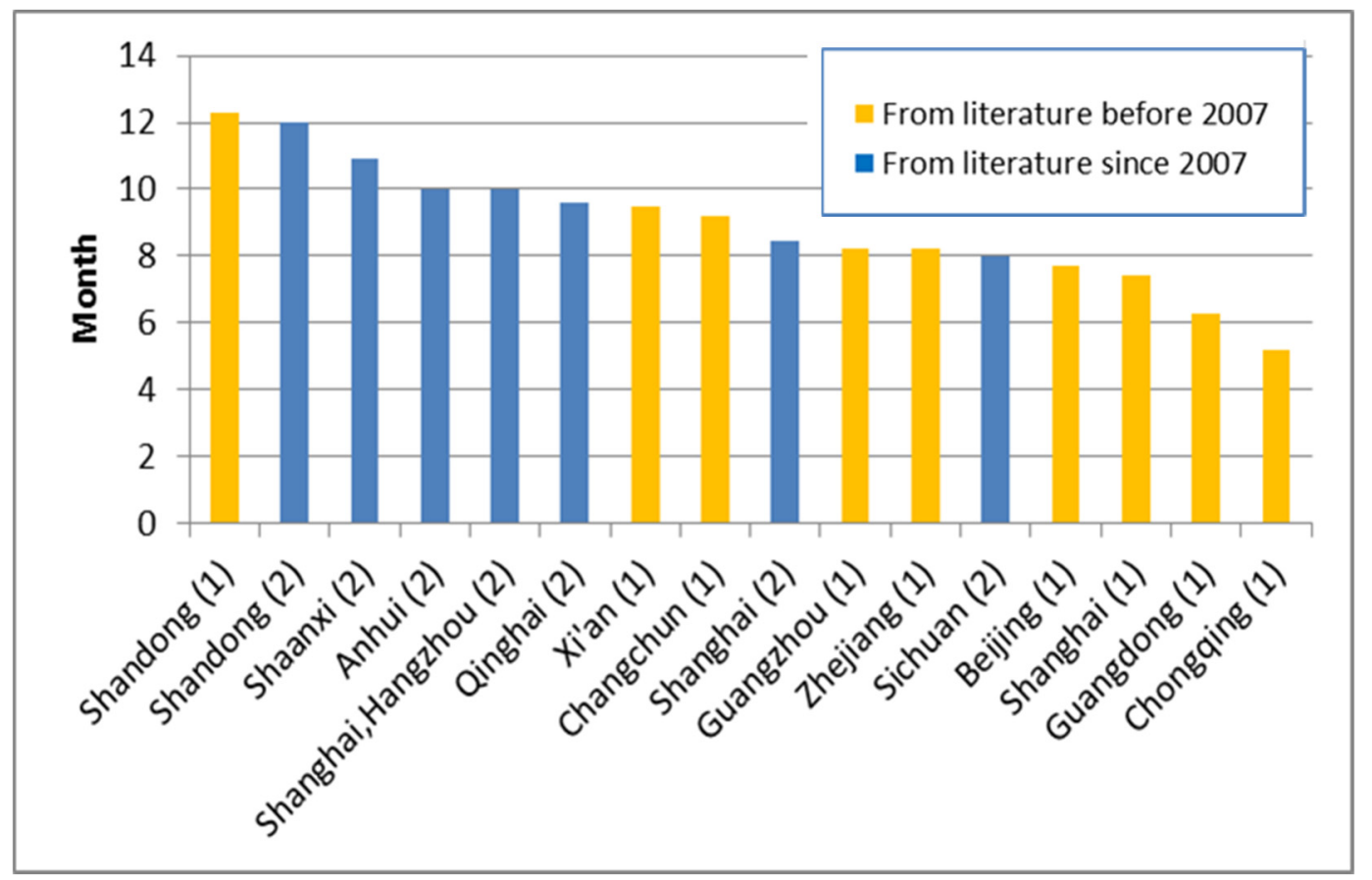

Figure 5. Comparison of length of breastfeeding in China before and after 2007.

A cross-sectional study $(n=635,2017)$ undertaken in four hospitals in Shandong province reported the longest mean duration of "any breastfeeding" of $12.0 \pm 0.82$ months. The mean "exclusive breastfeeding" duration was 4.01 months [73]. In this study, all mothers participating were nurses [73]. A cohort study in 17 cities from Shandong province $(n=1630)$ showed that the average duration of "exclusive breastfeeding" was $6.15 \pm 2.35$ months (mean \pm SD) in 2008-2013 [76]. Another cohort study at Lianyungang city, Jiangsu province $(n=383)$, indicated the average duration of "exclusive breastfeeding" was $5.98 \pm 4.43$ months (mean \pm SD) in 2013-2014 [78]. The studies from other cities showed that the length of "exclusive breastfeeding" were between 2.9 and 5.36 months $[44,75,77,80]$. The study in Shihezi city ( $n=200,2007-2008)$, Xinjiang province, showed the shortest duration of "exclusive breastfeeding" (0.92 month) [79].

The reasons for the increase in breastfeeding duration may include policy changes; the increase in maternity leave [81,82], institution of a "universal two-child policy" [83,84] and more breastfeeding promotion, including using mobile telephones to send educational messages regularly to mothers $[85,86]$. Maternity leave with full salary was extended from 90 days to 98 days in 2012 in China [81] and from 98 days to $128-180$ days in the majority provinces from 2016 when the "universal two-child policy" was implemented throughout the country [82]. Fathers have nursing leave (paternity leave) of 7 to 30 days with full salary since 2016 [82]. Employers provide mothers with one hour for breastfeeding during work time [81]. With the policy support, mothers and families have less financial burden and more time for breastfeeding [84].

The two-child policy has been gradually implemented in China since $2011[83,84,87]$. Couples have been allowed to have the second baby if both of them come from a single-child family since 2011, if at least one of them come from single-child family since 2013 and unconditionally since 2016 (the "universal two-child policy") [83,84]. Chinese citizens from ethnic groups are not subject to these limits. Studies showed that the second babies have longer breastfeeding duration (for both "any" and "exclusive breastfeeding") than the first [74,80]. A survey in Hangzhou and Shanghai in 235 multiparous mothers (2017) showed that both "exclusive breastfeeding" and "any breastfeeding" durations for the second baby were longer than for the first one [80]. "Exclusive breastfeeding" duration was 3.28 months for the first baby and 3.61 months for the second baby $(p<0.05)$; and "any breastfeeding" duration was 9.78 months for the first baby and 10.88 months for the second baby 
for $(p<0.01)$ [80]. Similarly, a retrospective study $(n=168)$ in Zhejiang province in 2017 found that "exclusive breastfeeding" duration and "any breastfeeding" duration of the second baby were longer than those of the first $(p<0$. 05) [74]. "Any breastfeeding" duration was $9.8 \pm 3.7$ months for the first baby and $11.6 \pm 4.6$ months for the second [74]. "Exclusive breastfeeding" duration was $2.9 \pm 2.4$ months for the first baby and $3.3 \pm 2.3$ months for the second [74]. The reason for the increase in breastfeeding duration of the second baby may associated with the mother's breastfeeding experience from the first baby, more involvement of fathers or more supportive breastfeeding policy [82].

In addition, breastfeeding education using the internet and mobile apps increased breastfeeding knowledge of mothers, fathers and their families $[65,72,88]$. A study in Shanghai found that receiving a weekly text message supporting breastfeeding significantly improved the "exclusive breastfeeding" rate (from $6.3 \%$ to $15.1 \%$ ) and increased the duration of "exclusive breastfeeding" assessed at six months (from 8.87 to 11.41 weeks) [85]. The use of mobile applications appears to be an effective way to support breastfeeding and increase "exclusive breastfeeding" rates [86]. Participating in "pregnancy school" (antenatal classes) was also associated with increased breastfeeding duration [80]. Other risk factors associated with breastfeeding duration included mother's education level (mothers with high education level were more likely to be employed and had shorter breastfeeding duration), grandparents' support (babies were breastfed for a shorter duration if grandparents helped to take care of them) and having breastfeeding difficulties (babies were less likely to be breastfed if their mother had breastfeeding difficulties) [80].

\subsection{Breastfeeding in Minority Areas or Groups}

In the Xinjiang Uygur Autonomous Region, the "exclusive breastfeeding" rate and "any breastfeeding" rates at one and a half months were $57.65 \%$ and $93.04 \%$, respectively, in the city of Yili in 2016 [89]. A cross-sectional study showed the "exclusive breastfeeding" rate at four months to be $63.03 \%$ in Tuoli between 2013 and 2015 [90]. In the Tibet Autonomous Region, "exclusive breastfeeding" and "any breastfeeding" rates at discharge were $22 \%$ and $75 \%$, respectively, in Lasa in 2016 [91]. Another survey in Sajia showed that "any breastfeeding" rates were 93.50\% at six months, declining to $52.03 \%$ at 12 months; and no-one was still "exclusively breastfeeding" at six months in 2013 [92].

In Yunnan province, a survey in Kunming showed that "exclusive breastfeeding" and "any breastfeeding" rates at four months were $48.52 \%$ and $79.50 \%$, respectively, in 2010 [93]. In Inner Mongolia, a cross-sectional study in Hailaer city in 2014-2015 showed that "exclusive breastfeeding" and "any breastfeeding" rates were $30.16 \%$ and $73.81 \%$, respectively, before six months [94]. In the Ningxia Hui Autonomous Region, the "any breastfeeding" rate at four months was $86.15 \%$ and the "exclusive breastfeeding" rate at four months was 62.44\% in 2009 [95]. In the Guangxi Zhuang Autonomous Region, the "exclusive breastfeeding" rate at 1.5 months was $64.18 \%$ in Nanning from 2010 to 2011 [96], and the rate at the same months was 73.33\% in Liuzhou in 2014 [97].

In summary, "exclusive breastfeeding" rates were lower in the minority areas. For example, "exclusive breastfeeding" rate at four months was $63.03 \%$ in Tuoli, $62.44 \%$ in Ningxia and $48.52 \%$ in Kunming $[90,93,95]$, and the "exclusive breastfeeding" rate at six month was $30.16 \%$ in Hailaer and $0 \%$ at Sajia $[92,94]$. "Any breastfeeding" rates were in a larger range. For example, the "any breastfeeding" rate at four months was $86.15 \%$ in Ningxia and 79.50\% in Kunming [93,95], and the "any breastfeeding" rate at six months was $93.50 \%$ at Sajia and $73.81 \%$ in Alaer $[92,94]$.

\subsection{Changes in Breastfeeding Rates in China from 2007 to 2017}

During the latest decade, a series of initiatives and measures have been taken to promote breastfeeding in China, including promoting the Baby Friendly Hospital Initiative, women and child health protection legislation, ten steps to successful breastfeeding, society support programs, breastfeeding education programs, continuous nursing and complementary food control $[98,99]$. The Baby Friendly Hospital Initiative is used to promote breastfeeding in China [6]. In 1992, 
only 21 state-owned hospitals in China had received baby friendly hospital initiative certification. By 2015, there were 7036 certified baby-friendly hospitals in China, and 66\% of births took place in baby-friendly hospitals [100].

The fourth national health service survey in 2008 showed that the "exclusive breastfeeding" rate at six months was $27.6 \%$ [101]; the rate increased to $58.5 \%$ in the fifth national health service survey in 2013 [102]. In Shanghai, after promulgation of "the Outline of Development of Chinese Women (2011-2020)", the "exclusive breastfeeding" rate of 3585 newborn infants in Jiangwan Community of Hongkou District in 2012-2016 was significantly higher than that of 3367 newborn infants in the same area in 2007-2011 (49.6\% vs. $26.9 \%, P<0.05)$ [98]. A quasi-experimental study $(n=16,867)$ throughout 14 provinces in Eastern, Central and Western China showed that "exclusive breastfeeding" rates at six months increased significantly from $42.96 \%$ in 2012 to $48.84 \%$ in 2015 after carrying out standard health management for children in these areas [103]. On the other hand, a retrospective study $(n=8673)$ in poverty-stricken areas from 13 provinces of China in 2007-2009 showed that "any breastfeeding" rates at six months decreased from $93.8 \%$ in 2007 and $93.5 \%$ in 2008 to $91.7 \%$ in 2009; and "full breastfeeding" rates at six months also declined slightly from $52.3 \%$ in 2007 to $43.4 \%$ in 2008 and $48.2 \%$ in 2009 [104].

Compared with the results in our previous literature review [6], the proportion of studies measuring breastfeeding rates, including "exclusive breastfeeding" and "any breastfeeding" rates, at six months has increased. This may be associated with the changes in breastfeeding targets set in the National Program of Action for Child Development in China. In the 1990s, the target was breastfeeding of $80 \%$ by 2000 and promoting "exclusive breastfeeding" to four or six months [6]. The target from 2001 to 2010 was a breastfeeding rate of $85 \%$ and timely introduction of complementary food [6]. The recent target from 2011 to 2020 is an "exclusive breastfeeding" rate of 50\% and over at the sixth month of life [5]. The new target is more specific than the previous targets [5].

The main problem found in this review of breastfeeding in China is the "exclusive breastfeeding" rates, which remain low (Figure 3). This is consistent with reports from other countries, with only an average of $38 \%$ of infants aged 0 to 6 months being exclusively breastfed globally [105]. The World Health Organization International Code of Marketing of Breastmilk Substitutes (the Code) is critical to protecting exclusive breastfeeding [105]. However, more effort needs to be made to ensure that the Code is followed everywhere in China. A study among 291 mothers with babies under 6 months old from six cities in China found that $40.2 \%$ of the mothers reported receiving free formula samples, violations of the Code, with $76.1 \%$ received the free samples in or near a hospital [106].

\subsection{Reasons for Discontinuing Breastfeeding or Exclusive Breastfeeding before Six Months in China}

Table 4 shows the reasons for discontinuing breastfeeding or introducing water, formula or other infant food before six months in nine cities (Shanghai [69,80,107], Hangzhou [80], Yuncheng [108], Shenzhen [71], Xining [50,51], Yongkang [29], Xi'an [50], Panzhihua [109] and Kunming [93]). in China. From three studies (in Xi'an and Xining, Shenzhen, and Kunming), the reasons for discontinuing "exclusive breastfeeding" were perceived breastmilk insufficiency (the first reason), mother's returning to work (the second reason), maternal and child illness (the third reason for Xi'an and Xining and Kunming) and concern about nutrition or available formula milk (the third reason for Shenzhen). From the seven studies in six cities (two studies in Shanghai), the reasons for discontinuing "any breastfeeding" were "perceived breastmilk insufficiency" (the most common reason for five cities except Shanghai) and "mother returning to work" (the first reason for Shanghai, the second reason for other four cities except Panzhihua). See Table 4 for more details. A survey in mothers of 0-3-year-old children in Guangzhou showed that $39.8 \%$ of mothers thought their breast milk supply was insufficient, $17.2 \%$ of them felt that breastfeeding should be stopped when babies grew to a certain month of age (the cut-off month was not mentioned) [35]. In a cross-sectional study in mothers with 0-2 years old children in Sajia, a county of the Tibet Autonomous Region, 19.6\% mothers thought that their babies should be weaned off breastfeeding [92]. In Shandong province, taking the night shift (accounted for $54.19 \%$ ) was the main reason for weaning breastfeeding among mothers who were nurses [73]. 
Table 4. Reasons for discontinuing breastfeeding in the first six months after birth.

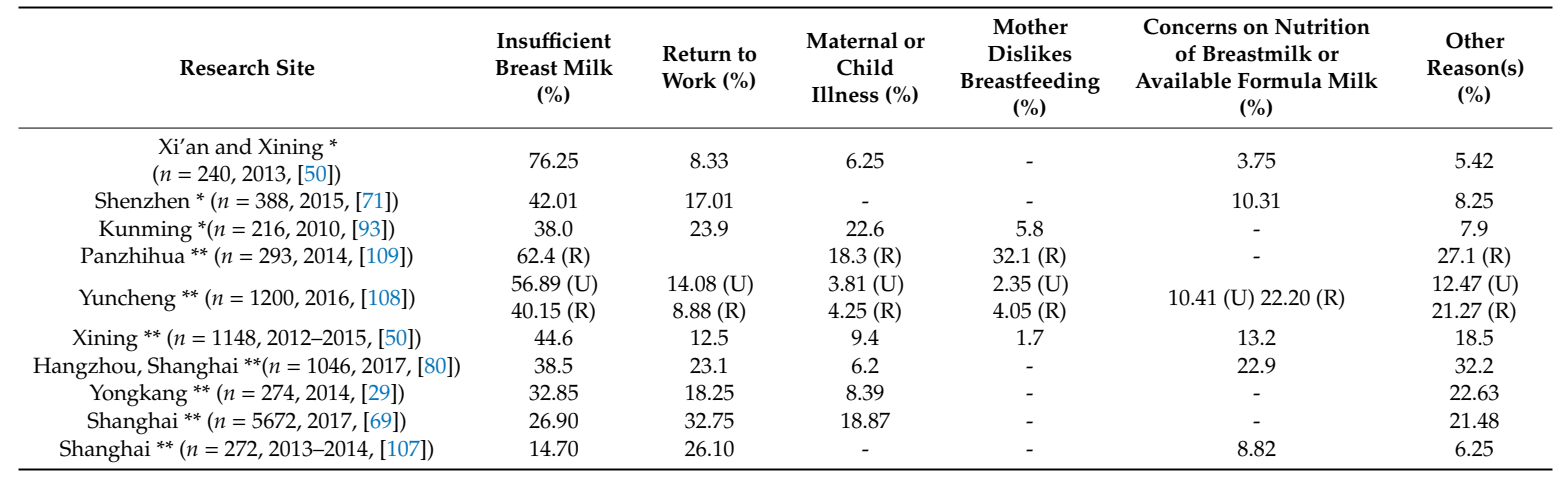

* Reasons for discontinuation of "exclusive breastfeeding". ${ }^{* *}$ Reasons for discontinuation of "any breastfeeding".

U: urban; R: rural.

The common reasons given for ceasing breastfeeding or "exclusive breastfeeding" before six months were: perceived breast milk insufficiency, mother returning to work and maternal or child illness. The three reasons were similar to those reported in a previous review [6]. Moreover, concern about nutrition or available formula milk was another common reason for discontinuing breastfeeding in recent years.

There are several limitations that need to be considered when interpreting the results of this literature review. We have endeavoured to find all relevant studies. However, there are only two extensive electronic data bases in the Chinese language. There may be some small regional journals that are not included in these databases. We have relied on the databases, our own knowledge of this field and consultations with colleagues. The study does not include several special areas of China, such as, Macao, Hong Kong and Taiwan. Any studies published in languages other than English or Chinese have not been included in this review. While the National Government and provincial centres for disease controls provide guidance on the measurement of breastfeeding and other public health parameters, there may be some variation in definitions used and methodology across the country. The comparability of studies across time periods in a vast country is a limitation that has to be considered in interpreting the data. The authors have presented the data as recorded in the published literature.

\section{Conclusions}

The mean duration of "any breastfeeding" in China appears to have increased by up to two months in the last decade. The proportion of mothers breastfeeding at four months has increased from $78 \%$ to $83 \%$. The second baby has a longer breastfeeding duration than the first. While breastfeeding statistics have improved, the exclusive breastfeeding rate is below the national goal. Breastfeeding education needs to emphasize the benefits of "exclusive breastfeeding" for six months and correct traditional perceptions. The findings of this review show the need to explore further factors that are associated with the early introduction of complementary food to babies and to provide more education on exclusive breastfeeding.

Author Contributions: Q.L. translated literature, analysed the results and wrote the manuscript. J.T. viewed and wrote the manuscript. F.X. analysed the results, wrote and revised the manuscript. C.B. wrote and revised the manuscript. All authors have read and agreed to the published version of the manuscript.

Funding: Funding for this project came from the Department of Science and Technology of Hebei, China, grant no. 142777106D. Further support came from the Department of Nursing of Chengde Medical University China.

Acknowledgments: We would like to thank the Department of Science and Technology of Hebei, China for funding this study under grant no. 142777106D. Further support came from the Department of Nursing of Chengde Medical University China; the School of Public Health of Curtin University Australia and the Data Analysis and Surgical Outcomes Unit of Royal North Shore Hospital of Sydney Australia.

Conflicts of Interest: The authors declare that there are no conflict of interest. 


\section{References}

1. Victoria, C.G. Effect of breastfeeding on infant and child mortality due to infectious diseases in less developed countries: A pooled analysis. WHO Collaborative Study Team on the Role of Breastfeeding on the Prevention of Infant Mortality. Lancet 2000, 355, 451-455.

2. Zhou, Y.; Chen, J.; Li, Q.; Huang, W.; Lan, H.; Jiang, H. Association between breastfeeding and breast cancer risk: Evidence from a meta-analysis. Breastfeed. Med. 2015, 10, 175-182. [CrossRef]

3. WHO. Breastfeeding. Available online: http://www.who.int/maternal_child_adolescent/topics/child/ nutrition/breastfeeding/en (accessed on 13 November 2018).

4. WHO. Global Nutrition Targets 2025: Policy, Brief and Series. Available online: http://www.who.int/nutrition/ publications/globaltargets2025_policybrief_overview/en/ (accessed on 13 November 2018).

5. China State Council. National Program of Action for Child Development in China (2011-2020); 2011-08-09; China People's Publishing House: Beijing, China, 2011; p. 14.

6. Xu, F.; Qiu, L.; Binns, C.W.; Liu, X. Breastfeeding in China: A review. Int. Breastfeed. J. 2009, 4, 6. [CrossRef]

7. Von Elm, E.; Altman, D.G.; Egger, M.; Pocock, S.J.; Gøtzsche, P.C.; Vandenbroucke, J.P. The Strengthening the Reporting of Observational Studies in Epidemiology (STROBE) Statement: Guidelines for reporting observational studies. Int. J. Surg. 2014, 12, 1495-1499. [CrossRef]

8. Zhao, J.; Zhao, Y.; Du, M.; Binns, C.W.; Lee, A.H. Does Caesarean Section Affect Breastfeeding Practices in China? A Systematic Review and Meta-Analysis. Matern. Child Health J. 2017, 21, 2008-2024. [CrossRef]

9. WHO. Infant and Young Child Feeding: A Tool for Assessing National Practices, Policies and Programmes. Available online: https://www.who.int/nutrition/topics/global_strategy/en/(accessed on 5 July 2020).

10. Shi, D.; Ma, L.; Ying, Q. A survey on breastfeeding among medical workers in Beijing. J. Hyg. Res. 2011, 40, 653-654. [CrossRef]

11. Zhao, M.; Liu, J.; Wang, Q. A survey of breastfeeding of twin babies. J. Nurs. Sci. 2015, 30, 29-32. [CrossRef]

12. Mei, H.; Zhai, J.; Luo, X. Promote exclusive breastfeeding by the application of forward control. Chin. J. Fam. Plan. Gynecol. 2017, 9, 45-48. [CrossRef]

13. Yu, C.; Binns, C.W.; Lee, A.H. Comparison of breastfeeding rates and health outcomes for infants receiving care from hospital outpatient clinic and community health centres in China. J. Child Health Care 2016, 20, 286-293. [CrossRef]

14. Li, Y.; Ma, J.; Lan, J.; Zhang, X. Analysis of breastfeeding rate and influence factors among 747 infants in one community. Ningxia Med. J. 2016, 38, 845-846. [CrossRef]

15. Liu, Y.; Fu, L. The status of exclusive breastfeeding for 0-6 month infants and the influence on physical development. Matern. Child Health Care China 2015, 30, 5045-5046. [CrossRef]

16. Zhang, X.; Pang, M.; Li, N. The investigation of the breastfeeding and its influencing factors. ShaanXi Med. Mag. 2015, 44, 1419-1420. [CrossRef]

17. LI, Q. The investigation and analysis on breastfeeding of 416 babies and mothers' dietary. Chin. J. Clin. Ration. Drug Use 2010, 3, 109-110. [CrossRef]

18. Qiu, H.; Wu, X.; Wu, J. Effect of Individualized Continuous Health Education on Improving the Method of the Breastfeeding of Uyghur Women. J. Nongken Med. 2017, 39, 347-349. [CrossRef]

19. Cui, X. Study of breastfeeding and 12 months social response. Matern. Child Health Care China 2016, 31, 513-516.

20. OuYang, F.; Yu, Z.; Wang, X.; Xi, T.; Liu, F. A survey on situation of breastfeeding among 0-6 month infants in urban villages. Chin. J. Gen. Pract. 2015, 13, 1125-1127. [CrossRef]

21. Jia, X.; Xu, J. Survey on breastfeeding among infants of 0-6 months. J. Community Med. 2014, 12, $12-14$.

22. Chen, R.; Yang, Y.; Tian, N.; Mai, W.; Li, A. The study of the breastfeeding and its influencing factors of primipara. Mod. Prev. Med. 2013, 40, 625-626.

23. Tang, L.; Lee, A.H.; Binns, C.W. Factors associated with breastfeeding duration: A prospective cohort study in Sichuan Province, China. World J. Pediatr WJP 2015, 11, 232-238. [CrossRef]

24. Gu, C.; Yan, S.; Li, Q.; Du, Y.; Wang, S.; Zhu, H. Follow-up survey of premature infants' breastfeeding. Chin. Rural Health Serv. Adm. 2010, 30, 577-579.

25. Li, J.; Li, Z. Investigation and analysis of breastfeeding practices in Mianyang urban area. Chongqing Med. 2009, 38, 2876-2877. [CrossRef] 
26. Fei, W.; Ying, Y. The impact of effective early sucking on breastfeeding for baby at 6 months. Chin. J. Child Health Care 2009, 17, 481-482.

27. Chen, Q.; Zhao, W.; Lin, Q.; Wang, E.; Zheng, S.; Chen, Y. The status quo of breastfeeding for baby with 6 months in Wenling city. China Rural Med. 2017, 24, 55-57. [CrossRef]

28. Lei, J.; Jin, H.; Ye, P.; HE, J.; Zhou, X.; Fu, W.; Liu, L. A study on the influencing factors of breastfeeding for 0-6 month infants. Zhengiiang Prev. Med. 2015, 27, 1115-1118. [CrossRef]

29. Wu, Y.; Qiu, L. An analysis on the status and influencing factors of breastfeeding among infants within six months of age. Zhengjiang Prev. Med. 2015, 27, 245-248. [CrossRef]

30. Jiao, Y.; Huang, S. The investigation and analysis of breastfeeding in kang county in Longnan region. Chin. Community Phys. Med. Prof. 2011, 13, 203. [CrossRef]

31. Xu, F.; Liu, X.; Binns, C.W.; Xiao, C.; Wu, J.; Lee, A.H. A decade of change in breastfeeding in China's far north-west. Int. Breastfeed. J. 2006, 1, 22. [CrossRef]

32. Xu, F.; Binns, C.; Zheng, S.; Wang, Y.; Zhao, Y.; Lee, A. Determinants of exclusive breastfeeding duration in Xinjiang, PR China. Asia Pac. J. Clin. Nutr. 2007, 16, 316-321. [CrossRef]

33. Gao, X. The Status and Influencing Factors of Breastfeeding in Poor Rural Areas of Western China. Master's Thesis, Shaanxi Normal University, Xi'an, Chian, 2017.

34. Huang, Y.; Zhang, W.; Fang, L. Duration of breastfeeding and its influencing factors in children from poor areas of Anhui. Chin. J. Child Health Care 2017, 25, 1150-1152. [CrossRef]

35. Wang, N.; Chen, Q. Study on Factors of Duration of Infants Breastfeeding: Based on Questionnaire Survey of Yuexiu District of Guangzhou City. Chin. J. Soc. Med. 2017, 34, 478-482. [CrossRef]

36. Wu, J.; Shao, B.; Huang, M.; Gu, L.; Li, M.; Jiang, S.; Mo, M.; Ximusiye, M.; Wang, J.; Jiang, W.; et al. The situation and influencing factors of breastfeeding in Zhoushan from 2002 to 2015. Chin. J. Dis. Control Prev. 2018, 22, 485-489. [CrossRef]

37. Gao, W. Analysis of breastfeeding and its influencing factors in Heping district of Tianjin. J. Qiqihar Univ. Med. 2016, 37, 3068-3070.

38. Wang, J. A survey on breastfeeding of infants at 0-6 months. Nei Mong. J. Tradit. Chin. Med. 2014, 33, 60. [CrossRef]

39. Tang, J. Influencing factors and intervention measures of infant pure breast feeding compliance. China Pract. Med. 2016, 11, 191-193. [CrossRef]

40. Sun, S.; Zhang, Z.; Wan, H. Survey of influencing factors of postpartum breastfeeding. Chin. Nurs. Res. 2016, 30, 2626-2629. [CrossRef]

41. Zhang, Y.; Jiang, H. The analysis on the status and influencing factors of the breast-feeding postpartum in 42 days. Today Nurse 2017, 6, 35-37.

42. Cheng, G. Regulation of the Impact of Children's Health Management on Children's 6-month Exclusive Breastfeeding Rate. Syst. Med. 2018, 3, 184-185. [CrossRef]

43. Zhang, Q.; Shi, Y. The analysis of influencing factors of breastfeeding rates among 612 women. Matern. Child Health Care China 2013, 28, 5095-5096. [CrossRef]

44. Fu, Y. Investigate the Affect of Post-natal Care Intervention on Breastfeeding Rates and Duration. China Health Stand. Manag. 2015, 6, 256-258. [CrossRef]

45. Zhou, R. The related factors and intervention for Breastfeeding and its compliance. Med. J. Present Clin. 2017, 30, 3414-3418. [CrossRef]

46. Deng, Y. A Study on Breastfeeding Self-Efficacy and its Influencing Factors in Postpartum Women from 0 to 6 Months in Wuhan. Mater's Thesis, Wuhan Polytechnic University, WuHan, China, 2017.

47. Li, G.; Chen, X.; Pan, Y.; Luo, J.; Fan, X. Survey on the relationship between breastfeeding and anemia in 6-month-old child of flower town in Guangzhou. J. Baotou Med. Coll. 2016, 32, 14-16. [CrossRef]

48. Chen, Z.; Wang, S.; Zhai, C. The effect of postpartum extended care on promoting breastfeeding practice. Acad. J. Guangzhou Med. Univ. 2015, 43, 119-120. [CrossRef]

49. Zhou, H.; Kuang, Y.; Hou, D.; Xie, D.; Zhang, J. The status quo of breastfeeding and its influencing factors in Changsha. Pract. Prev. Med. 2017, 24, 210-212. [CrossRef]

50. Ma, Y.; Xu, D.; Wang, L.; Wang, Y. Breast feeding studies on Hui nationality infants in Xining and Xian areas. Chin. J. Child Health Care 2014, 22, 871-873. [CrossRef]

51. Lv, F. A survey on breastfeeding of infants at 6 months age in Xining. Qinghai Med. J. 2016, 46, 59-60. 
52. Feng, S.; Liu, Y.; Guo, J.; LI, X.; Zhao, L.; Liu, B.; Zhang, X.; Liu, H. Analysis on neonatal feeding status and influencing factors in Changchun city. Matern. Child Health Care China 2015, 30, 5844-5846. [CrossRef]

53. Wang, Q.; Ge, M.; Shao, J. A survey on breastfeeding of infants in rural and urban areas in Jilin province in 2007-2010. Matern. Child Health Care China 2012, 27, 4255-4256.

54. Chen, W.; Liang, X.; Zhou, J. A survey on exclusive breastfeeding of infants before 6 months age and its influencing factors in Nanchang. Chongqing Med. 2014, 43, 3399-3401. [CrossRef]

55. Xu, W.; Lei, J.; Zhou, Q. A survey on primipara's breastfeeding confidence and its influencing factors. J. Tradit. Chin. Med. Manag. 2018, 26, 14-17. [CrossRef]

56. Liu, L.; Hu, J.; Jin, H.; LEI, J. Investigation and related factors analysis of breast feeding of infants under 6 months in Lishui. Chin. J. Child Health Care 2014, 22, 1092-1095. [CrossRef]

57. Zhao, F. Status and related factors of exclusive breastfeeding of infants within 6 months in a certain area. Prev. Med. 2018, 3, 105-106. [CrossRef]

58. Wang, N. A survey on breastfeeding at 4-6 months among primipara. J. Heze Med. Coll. 2015, $27,85-87$. [CrossRef]

59. Guo, L. The Study of Impact on Social and Psychological Factors in Breastfeeding. Master's Thesis, Shanxi Medical university, Taiyuan, China, 2010.

60. Yin, X.; Gui, J.; Wang, H.; Huang, X.; Zhou, M.; Zhu, P. Follow-up survey of risk factors for early cessation of breastfeeding in early infancy. Chin. J. Neonatol. 2012, 27, 148-152. [CrossRef]

61. Cai, C.; Hao, J.; Tao, F.; Zhang, Y.; Wang, D.; Zhu, P.; Su, P.; Sun, L. Dietary Patterns in Pregnancy, Breastfeeding and Mental Development in Infants: A Population-Based Cohort Study. J. Anhui Med. Univ. 2013, 48, 634-638.

62. Zhang, Z. The related factors and prevention countermeasures for breastfeeding compliance before 6 months. Contemp. Med. 2017, 23, 32-33. [CrossRef]

63. Zhu, D. The influence of postpartum visit on exclusive breastfeeding. J. Taishan Med. College 2014, 35, 1190-1191. [CrossRef]

64. Zhang, Y.; Wang, H.; Luo, Q.; Zhang, L.; Wang, S.; Yao, L. The exclusive breastfeeding of newborns and its influencing factors. Chin. J. Child Health Care 2012, 20, 507-509.

65. Yang, M.; Liu, N.; Yang, C.; Zheng, X.; Xiong, C.; YANG, S.; Du, Y.; Zhang, J. Analysis on the effect factors of pure breastfeeding among the rural infants in five provinces of Western China. Matern. Child Health Care China 2012, 27, 1035-1037.

66. Guo, S.; Fu, X.; Scherpbier, R.W.; Wang, Y.; Zhou, H.; Wang, X.; Hipgrave, D.B. Breastfeeding rates in central and western China in 2010: Implications for child and population health. Bull. World Health Org. 2013, 91, 322-331. [CrossRef] [PubMed]

67. Duan, Y.; Yang, Z.; Lai, J.; Yu, D.; Chang, S.; Pang, X.; Jiang, S.; Zhang, H.; Bi, Y.; Wang, J.; et al. Exclusive Breastfeeding Rate and Complementary Feeding Indicators in China: A National Representative Survey in 2013. Nutrients 2018, 10. [CrossRef]

68. Zhang, L.; Feng, S.; Wen, F. Analysis of the status and influencing factors of breastfeeding among infants within six months of ages in Haidian neighbourhood of Haidian district of Beijing. Chin. J. Child Health Care 2016, 24, 1081-1083. [CrossRef]

69. Li, W.; Du, L.; Hu, S.; Zhu, L. Survey on knowledge, attitude, belief and practice of exclusive breastfeeding among mothers of infants aged below 6 months in Shanghai. Chin. J. Dis. Control Prev. 2018, 22, 490-493. [CrossRef]

70. Su, S.; Qu, W.; Ni, B.; Sun, J. Status quo and influencing factors of breastfeeding among infants aged 6-month and elder in Dalian municipality. Chin. J. Public Health 2017, 33, 792-795. [CrossRef]

71. Wu, X.; Sun, X.; He, S. Analysis of the status and influencing factors of the exclusive breastfeeding among six months infants. Chin. J. Child Health Care 2017, 25, 90-93. [CrossRef]

72. Yang, S.; Hong, Q.; Sun, X.; Hao, Y.; Deng, Y.; Duan, S. Analysis of the status and influencing factors of breastfeeding in Anhui province. Chin. J. Child Health Care 2017, 25, 1152-1155. [CrossRef]

73. Wang, F. The Status and Influencing Factors Analysis of the Postpartum Nurse' Breastfeeding in Third-Grade Class-A Hospitals. Master's Thesis, Shandong University, Jinan, China, 2018.

74. Li, C.R.; Du, Y.P. Quantitative analysis of breastfeeding and maternal and infant health care conditions in different fetal times of multiparas under the two-child policy. Chin. Gen. Pract. 2017, 20, 3659-3664. [CrossRef] 
75. Huang, Y.; He, P.; Huang, B.; Wang, F. Analysis on factors that may influence working mother's breastfeeding practice. Chin. J. Child Health Care 2014, 22, 142-145.

76. Liu, W.; Li, S. Duration of exclusive breastfeeding and its influence factors in Shandong. Chin. J. Child Health Care 2016, 24, 410-412. [CrossRef]

77. Hua, J.; Wu, Z.; Deng, W.; Duan, C.; Lei, P.; Gu, G.; Yan, H.; Yao, F.; Wang, Y. Factors influencing exclusive breastfeeding in the rural areas of mid-west China. Chin. J. Child Health Care 2010, 25, 3229-3232.

78. Zhang, W. The impact of Continuous care on maternal skills and exclusive breastfeeding for women within 6 months after delivering. Today Nurse 2017, 74-75.

79. Liu, P.; Wang, Y.; Yu, P.; Le, Y.; Tang, L.; Xu, F. Influence of Breastfeeding Handbook on Exclusive Breastfeeding within First Six Months. J. Appl. Clin. Pediatr. 2010, 25, 366-367.

80. Li, C. Study on the Implementation and Influencing Factors of Breastfeeding and Health Care Service in Eastern China after the Second-Child Policy. Master's Thesis, Zhejiang University, Hangzhou, China, 2018.

81. Chinese Government. Special Regulation for Female Employee Protection. Available online: http://www. gov.cn/flfg/2012-05/07/content_2131582.htm (accessed on 8 September 2020).

82. Baidu Encylopedia. Maternity Leave. Available online: https://baike.baidu.com/item/\%E4\%BA\%A7\%E5\% 81\%87/9380360?fr=aladdin (accessed on 14 September 2020).

83. Chinese Government. Modification of Population and Family Planning Law. Available online: http: //www.npc.gov.cn/wxzl/gongbao/2016-02/26/content_1987077.htm (accessed on 14 September 2020).

84. Baidu Encyclopedia. Second-Child Policy. Available online: https://baike.baidu.com/item/\%E4\%BA $\% 8 C \%$ E5\%AD \%A9\%E6\%94\%BF\%E7\%AD\%96/18761690?fromtitle=\%E4\%BA \%8C\%E8\%83\%8E\%E\%94\%BF\% E7\%AD\%96\&fromid $=4671776$ (accessed on 14 September 2020).

85. Jiang, H.; Li, M.; Wen, L.M.; Hu, Q.; Yang, D.; He, G.; Baur, L.A.; Dibley, M.J.; Qian, X. Effect of short message service on infant feeding practice: Findings from a community-based study in Shanghai, China. JAMA Pediatr. 2014, 168, 471-478. [CrossRef]

86. Doan, T.T.D.; Binns, C.; Pham, N.M.; Zhao, Y.; Dinh, T.P.H.; Bui, T.T.H.; Tran, T.C.; Nguyen, X.H.; Giglia, R.; $\mathrm{Xu}$, F.; et al. Improving Breastfeeding by Empowering Mothers in Vietnam: A Randomised Controlled Trial of a Mobile App. Int. J. Environ. Res. Public Health 2020, 17, 5552. [CrossRef] [PubMed]

87. Chinese Government. The Law of Population and Family Planning. Available online: http://www.gov.cn/ banshi/2005-08/21/content_25059.htm (accessed on 14 September 2020).

88. China Development Research Founation. Report of Factors Associated with Breastfeeding in China (2017). Available online: https://cdrf.org.cn/jjhdt/4853.jhtml (accessed on 15 September 2020).

89. Feng, L. Clinical Research of Compliance Related Influence Factors of Women Breastfeeding in Ili Hospital. XinJiang Med. J. 2016, 46, 1307-1309.

90. Ma, X. A survey on breastfeeding and its influencing factors in Tuoli county. World Latest Med. Inf. Electron. Vers. 2017, 17, 150-151.

91. Huang, Y.; Cheng, Z. A survey on breastfeeding at discharge among 200 women. Tibet. Med. J. 2017, 38, 58-60.

92. Pubu, Q. The investigation on breastfeeding in 0-2 years old infant in Xizang. Xizang Med. 2017, 38, 52-53+49.

93. Shu, Y. Status Quo and analysis for exclusive breastfeeding in 4 communities in Dongchuan district. Chin. Community Dr. 2011, 13, 306-307.

94. Qi, M. Status Quo and analysis for breastfeeding in Hailaer area. Women's Health Res. 2017, 8, 50-51. [CrossRef]

95. Yang, Y. The analysis for breastfeeding and complementary food among infants at 0-4 months. J. HeBei United Univ. 2010, 12, 360-361. [CrossRef]

96. Ma, M. The analysis for influencing factors of exclusive breastfeeding among infants during the puerperium. Guaugxi Med. J. 2012, 34, 1704-1705.

97. Wu, Y. Effect of pregnancy health education on delivery modes and exclusive breastfeeding. Popul. Sci. Technol. 2015, 17, 73-75.

98. Wei, M. The impact of "the Outline of Development of Chinese Women (2011-2020)" on neonatal exclusive breastfeeding in the community. Shanghai Med. 2018, 39, 61-62. [CrossRef]

99. Yu, T.; Ye, M.; Dong, H.; Jiang, J. Influencial factors and nursing for breastfeeding behaviors of primiparas. J. Clin. Pathol. Res. 2018, 38, 1573-1578. 
100. UNICEF; WHO. Country Experiences with Baby-Friendly Hospital Initiative, Compendium Case Studies from around World; UNICEF: New York, NY, USA, 2017; p. 61.

101. Center for Health Statistics and Information NHPC. An Analysis Report National Health Services Survey in China, 2008; China Union Medical University Press: Beijing, China, 2009.

102. Center for Health Statistics and Information NHPC. The Fifth National Health Service Report, 2013; China Union Medical University Press: Beijing, China, 2015.

103. Yang, H.; Yin, D.; Xiao, F.; Li, R.; Xin, Q.; Wang, L.; Zheng, X.; Yin, T.; Liu, X.; Chen, B. The assessment of impact of Standard health management for children at 6 months on exclusive breastfeeding rate. Matern. Child Health Care China 2016, 31, 5066-5069. [CrossRef]

104. Qian, X.; Liu, A.; Yu, W.; Jiang, F.; Zhao, L. Analysis on the status and factors influencing the breastfeeding of children under 2 years of age in poverty stricken areas of China in 2007-2009. J. Hyg. Res. 2012, 41, 56-59.

105. Robinson, H.; Buccini, G.; Curry, L.; Perez-Escamilla, R. The World Health Organization Code and exclusive breastfeeding in China, India, and Vietnam. Matern. Child Nutr. 2019, 15, e12685. [CrossRef] [PubMed]

106. Liu, A.; Dai, Y.; Xie, X.; Chen, L. Implementation of international code of marketing breast-milk substitutes in China. Breastfeed. Med. 2014, 9, 467-472. [CrossRef]

107. Zhu, Y.; Wan, H. Influencing factors of pure breastfeeding behavior at postpartum 4 months on the basis on planned behavior theory. Chin. Nurs. Res. 2016, 30, 3051-3053. [CrossRef]

108. Zhao, Z.; Li, X.; Ma, P.; Yang, Z. The investigation of early exclusive breastfeeding after delivery among rural migrant women and urban women in the city of Yuncheng. Matern. Child Health Care China 2017, 32, 3016-3018. [CrossRef]

109. Li, R.; Chen, Y.; Chen, K.; Wei, X.; Zhao, J. Status and the influence factors of the exclusive breast feeding in Panzhihua east rural areas. Chin. J. Child Health Care 2017, 25, 627-630. [CrossRef]

Publisher's Note: MDPI stays neutral with regard to jurisdictional claims in published maps and institutional affiliations. 